\section{Differential diagnosis of POLG}

\section{related disorders: What to keep in mind when multiorgan system is \\ involved?}

\author{
Rajib Dutta* \\ MD, Neurology, India
}

\section{Abstract}

Mitochondrial and lysosomal dysfunction accounts for a large group of inherited metabolic disorders most of which are due to a dysfunctional mitochondrial respiratory chain (MRC) leading to deficient energy production and defects in phagocytosis in endosomal-lysosomal pathway respectively. MRC function depends on the coordinated expression of both nuclear (nDNA) and mitochondrial (mtDNA) genomes. Thus, mitochondrial diseases can be caused by genetic defects in either the mitochondrial or the nuclear genome, or in the cross-talk between the two. The mitochondrial DNA depletion syndromes (MDSs) are a clinically heterogeneous group of disorders with an autosomal recessive pattern of inheritance that have onset in infancy or early childhood and are characterized by a reduced number of copies of mtDNA in affected tissues and organs. In this review article, we summarized the spectrum of mtDNA depletion disorders along with minor learning of lysosomal storage diseases. This current article offers a perspective on the role of genetics in medical practice and how this role may evolve over the next several years.

\section{Introduction}

The polymerase gamma (POLG) gene (POLG1) is located on the long arm of chromosome 15 and encodes for alpha subunit DNA polymerase $\gamma$, while the POLG2 gene, located on the long arm of chromosome 17, encodes for its catalytic accessory subunit. Pol $\gamma$ functions in mitochondria. Uncontrolled mtDNA defects may result from damage to either of these two genes causing variable phenotypic features across ones' lifespan [1-3].

Mutations in POLG can cause early childhood mitochondrial DNA (mtDNA) depletion syndromes or later- onset syndromes arising from mtDNA deletions. It is very rare with an incidence of approximately 1 in 50000 people [4]. In several cases a family history of mitochondrial disorder is not found [1,2]. Mutations in POLG1 are inherited in autosomal recessive pattern whereas mutations in POLG2 can only lead to autosomal dominant progressive external ophthalmoplegia [1-3].

It ranges from myocerebrohepatopathy spectrum in infancy to parkinsonism in elderly. Six most common disorders are Alpers-huttenlocher syndrome (AHS), childhood myocerebrohepatopathy spectrum (MCHS), myoclonic

\begin{abstract}
More Information
*Address for Correspondence: Rajib Dutta MD, Neurology, India,

Email: rajibdutta808@gmail.com

Submitted: May 26, 2021

Approved: June 07, 2021

Published: June 09, 2021

How to cite this article: Dutta R. Differentia diagnosis of POLG related disorders: What to keep in mind when multiorgan system is involved? J Neurosci Neurol Disord. 2021; 5: 048-054.
\end{abstract}

DOI: 10.29328/journal.jnnd.1001050

ORCiD: orcid.org/0000-0002-6129-1038

Copyright: (C 2021 Dutta R. This is an open access article distributed under the Creative Commons Attribution License, which permits unrestricted use, distribution, and reproduction in any medium, provided the original work is properly cited.

Keywords: Mitochondria; lysosome; mtDNA; Metabolic; mtDNA depletion

Abbreviations: MERRF: Myoclonic Epilepsy with Ragged Red Fibers; NADH: Nicotinamide Adenine Dinucleotide Hydroxygenase; TBC: Tre2-Bub2-Cdc16 (TBC) Domain-Containing RAB-Specific Gtpase-Activating Protein; DNA: Deoxyribonucleic Acid; FBX: F-Box and Leucine-Rich Repeat Protein; MPV: Mitochondrial Inner Membrane Protein; SUCLA2: Succinate-Coa Ligase ADP-Forming Subunit Beta; SUCLG1: Succinate-Coa Ligase GDP/ADP-Forming Subunit Alpha; RRM2B:Ribonucleotide Reductase Regulatory TP53 Inducible Subunit M2B;TK2:Thymidine Kinase 2; MGME: Mitochondrial Genome Maintenance Exonuclease; SLC: Solute Carrier Family; BCS1L: BCS1 Homolog, UbiquinolCytochrome C Reductase Complex Chaperone; SC01: Synthesis Of Cytochrome C Oxidase 1; CLN1:Neuronal Ceroid Lipofuscinosis-1; CLN2:Neuronal Ceroid Lipofuscinosis-2; MELAS: Mitochondrial Myopathy, Encephalopathy, Lactic Acidosis, and Stroke

Check for updates

OPEN ACCESS

epilepsy myopathy sensory ataxia (MEMSA), ataxia neuropathy spectrum (ANS), autosomal recessive progressive external ophthalmoplegia (arPE0), autosomal dominant progressive external ophthalmoplegia (adPEO) [5]. Apart from the abovementioned disorders, there are several other diseases that are associated with mtDNA depletion with multiorgan presentation.

\section{Disorders to consider}

1) Epilepsia partialis continua (EPC): It is seen in 
Alpers-Huttenlocher syndrome (AHS) [6], and also can result from structural brain lesions (e.g., stroke, neoplasia, cortical dysplasia, traumatic lesion etc.). EPC has also been described in individuals with COQ8A-related primary coenzyme Q10 deficiency [7], NADH coenzyme Q reductase deficiency [8], MERRF, Leigh syndrome, and nonketotic hyperglycemia [9]. Recently, biallelic TBC1D24 pathogenic variants were identified in an individual with EPC [10].

2) Mitochondrial DNA Depletion (MDD) Disorders: MDD disorders may affect either a specific tissue (most commonly muscle or liver) or multiple organs, including the heart, brain, and kidney. MDD disorders need to be distinguished from the disorders of mtDNA mutation, duplication, or deletion. Mitochondrial DNA depletion syndromes, a genetically and clinically heterogeneous group of autosomal recessive disorders, are characterized by a severe reduction in mtDNA content leading to impaired energy production in affected tissues and organs.

Mitochondrial DNA depletion syndromes occur as a result of defects in mtDNA maintenance caused by pathogenic variants in nuclear genes that function in either mitochondrial nucleotide synthesis (e.g., TK2, SUCLA2, SUCLG1, RRM2B, DGUOK, and TYMP) or mtDNA replication(e.g., POLG and TWNK) $[11,12]$. Mitochondrial DNA depletion syndromes are phenotypically classified into myopathic, encephalomyopathic, hepatocerebral, and neurogastrointestinal forms [13].

Myopathic forms present in infancy or early childhood with hypotonia, proximal muscle weakness, dysarthria, feeding difficulty, and failure to thrive. Cognition is usually spared in this prototype. Typically, there is rapid progression of muscle weakness with respiratory failure and death within a few years of onset. Genes associated with this form are TK2 [14], RRM2B (p53R2) [15], DGUOK (dGK) [16].

Encephalomyopathic mtDNA depletion syndromes usually presents in infancy with hypotonia, muscle weakness, psychomotor delay, sensorineural hearing impairment, lactic acidosis, and neurological dysfunction. Closely associated genes are RRM2B (p53R2) [17], TK2 [18], SUCLA2 [19], SUCLG1 [20].

Hepatocerebral forms present in neonatal and early childhood associated with hepatic dysfunction, psychomotor delay, hypotonia, lactic acidosis, nystagmus, neurological dysfunction. Genes associated are C10orf2 (Twinkle/PEO1) [21], DGUOK (dGK) [22], MPV17 (SYM1) [23], TK2 [24].

Neurogastrointestinal forms, the prototype of which is mitochondrial neurogastrointestinal encephalopathy (MNGIE) disease, may present in adolescence to early adulthood with progressive gastrointestinal dysmotility, cachexia, and peripheral neuropathy, ptosis, and neurological dysfunction. Genes associated are TYMP (ECGF1) [25], RRM2B (p53R2) [26], a) Deoxyguanosine kinase deficiency (DGUOK deficiency): The two forms of DGUOK deficiency are a hepatocerebral mtDNA depletion syndrome (multisystem disease in neonates) and isolated hepatic disease later in infancy or childhood. The majority of affected individuals have the multisystem illness with hepatic disease (jaundice, cholestasis, and elevated transaminases) and neurologic manifestations (hypotonia, nystagmus, and psychomotor retardation) evident within weeks of birth. In contrast to AHS caused by POLG pathogenic variants, DGUOK deficiency is not characterized by seizures or brain imaging abnormalities [27].

Those with isolated liver disease may also have renal involvement and some later develop mild hypotonia. Progressive hepatic disease is the most common cause of death in both forms. Reduced mtDNA copy number in liver or muscle can be used to confirm mtDNA depletion. Molecular genetic testing of DGUOK is necessary to establish the specific diagnosis of DGUOK deficiency [22].

b) MPV17-related hepatocerebral mtDNA depletion syndrome (Navajo neurohepatopathy): It is characterized by liver failure, severe sensory neuropathy, corneal anesthesia and scarring, cerebral leukoencephalopathy, failure to thrive, and metabolic acidosis. Homozygosity for the MPV17 pathogenic variant NP_002428.1: p. Arg50Gln (NM_002437.4: c. $149 \mathrm{G}>\mathrm{A}$ ) is associated with Navajo neurohepatopathy, a mtDNA depletion syndrome displaying hepatic failure early in life, prevalent in the Navajo tribes in the southwestern United States and Mexico [28-30].

c) SUCLA2-related mtDNA depletion syndrome, encephalomyopathic form with methylmalonic aciduria: SUCLA2-related mitochondrial DNA (mtDNA) depletion syndrome, encephalomyopathic form with methylmalonic aciduria is characterized by onset of the following features in infancy or childhood (median age of onset 2 months; range of onset birth to 6 years): psychomotor retardation, hypotonia, dystonia, muscular atrophy, sensorineural hearing impairment, postnatal growth retardation, and feeding difficulties.

Other, less frequent features include distinctive facial features, contractures, kyphoscoliosis, gastroesophageal reflux, ptosis, choreoathetosis, ophthalmoplegia, and epilepsy (infantile spasms or generalized convulsions). The median survival is 20 years; approximately $30 \%$ of affected individuals succumb during childhood. Affected individuals may have hyperintensities in the basal ganglia, cerebral atrophy, and leukoencephalopathy on head MRI. Elevation of methylmalonic acid (MMA) in the urine and plasma is found in a vast majority of affected individuals, although at levels that are far below those typically seen in individuals with classic methylmalonic aciduria [31].

d) FBXL4-related encephalomyopathic mtDNA depletion syndrome: It is a multisystem disorder 
characterized primarily by congenital or early-onset lactic acidosis and growth failure, feeding difficulty, hypotonia, and global developmental delay. Other neurologic manifestations can include seizures, movement disorders, ataxia, autonomic dysfunction, and stroke-like episodes. All affected individuals alive at the time they were reported (median age: 3.5 years), demonstrated significant global developmental delay.

Other findings can involve the heart (hypertrophic cardiomyopathy, congenital heart malformations, arrhythmias), liver (mildly elevated transaminases), eyes (cataract, strabismus, nystagmus, optic atrophy), hearing (sensorineural hearing loss), and bone marrow (neutropenia, lymphopenia). Survival varies; the median age of reported deaths was two years (range 2 days - 75 months), although surviving individuals as old as 36 years have been reported. To date FBXL4-related mtDNA depletion syndrome has been reported in 50 individuals [32].

e) SUCLG1-related mtDNA depletion syndrome, encephalomyopathic form with methylmalonic aciduria: It is characterized in the majority of affected newborns by hypotonia, muscle atrophy, feeding difficulties, and lactic acidosis. Affected infants commonly manifest developmental delay, cognitive impairment, growth retardation, failure to thrive, hepatopathy, sensorineural hearing impairment, dystonia, and hypertonia.

Notable findings in some affected individuals include hypertrophic cardiomyopathy, epilepsy, myoclonus, microcephaly, sleep disturbance, rhabdomyolysis, contractures, hypothermia, and/or hypoglycemia. Life span is shortened, with median survival of 20 months. The phenotype may be indistinguishable from SUCLA2-related mtDNA depletion syndrome, encephalomyopathic form, with mild methylmalonic aciduria. Affected individuals have urinary excretion of MMA, combined respiratory chain enzyme deficiency, and mtDNA depletion [33].

f) RRM2B-related mitochondrial disease: Mutation of RRM2B has been associated with severe muscle mtDNA depletion in several families. This disorder manifests as severe encephalopathy, myopathy with persistent lactic acidosis, hypotonia, renal tubular defects, seizures, and diarrhea. Death has been reported to occur by age four months, but affected individuals have demonstrated longer survival [34].

g) Mitochondrial neuro-gastrointestinal encephalopathy (MNGIE): It is characterized by progressive gastrointestinal dysmotility (manifesting as early satiety, nausea, dysphagia, gastroesophageal reflux, postprandial emesis, episodic abdominal pain and/or distention, and diarrhea), cachexia, ptosis/ophthalmoplegia or ophthalmoparesis, leukoencephalopathy, demyelinating peripheral neuropathy, and symmetric and distal weakness more prominently affecting the lower extremities.
The order in which manifestations appear is unpredictable. Onset is usually between the first and fifth decade in about $60 \%$ of individuals and symptoms begin before 20 years. The diagnosis of MNGIE disease can be established in a proband by detection of one of the following: biallelic pathogenic variants in TYMP; markedly reduced levels of thymidine phosphorylase enzyme activity; and elevated plasma concentrations of thymidine and deoxy-uridine [35-38].

h) TK2-related mtDNA depletion syndrome: Mitochondrial myopathy with mtDNA depletion is caused by pathogenic variants in TK2. The clinical spectrum of TK2 mutations is not limited to severe infantile myopathy with motor regression and early death but includes spinal muscular atrophy type 3-like presentation, rigid spine syndrome, and subacute myopathy without motor regression and with longer survival [39-41].

3) MGME1-related mtDNA depletion syndrome 11: It is caused by biallelic pathogenic variants in MGME1. Individuals present between ages 10 and 36 years with ptosis, followed by mild PEO, diffuse skeletal muscle wasting and myopathy, profound emaciation, and respiratory failure. Intellectual disability was found in only one family of three affected individuals $[42,43]$.

4) SLC25A4-related mtDNA depletion syndrome 12B, cardiomyopathic type: It is caused by biallelic pathogenic variants in SLC25A4. Affected individuals may present with the SANDO phenotype which may include sensory ataxic neuropathy, dysarthria, and ophthalmoparesis [40].

\section{5) Other disorders to consider:}

a) Leigh syndrome: It is a progressive neurodegenerative disorder characterized by hypotonia, spasticity, dystonia, muscle weakness, hypo- or hyperreflexia, seizures, movement disorders, cerebellar ataxia, and peripheral neuropathy. In individuals with Leigh syndrome, MRI changes most often occur initially in the brain stem and the gliosis "migrates" over time to involve the deep gray masses and cortex, whereas in AHS the initial lesions form in the cerebral cortex (usually the occipital lobes), followed by the cerebellum, basal ganglia, thalamus, and brain stem [45].

b) Autosomal dominant progressive external ophthalmoplegia: It is caused by pathogenic variants in DGUOK, DNA2, OPA1, POLG2, RNASEH1, RRM2B, SLC25A4, TK2, or TWNK genes. Individuals with this disease may present with variable degrees of sensorineural hearing loss, generalized myopathy, depression, ataxia, parkinsonism, axonal neuropathy, cataracts, and hypogonadism [46,47].

c) Oculopharyngeal muscular dystrophy (OPMD): Disease onset is usually after 45 years and may result in progressive ptosis and bulbar dysfunction causing swallowing difficulty. This disorder may mimic the clinical manifestations 
of the POLG-related disorders in which PEO is the predominant feature. OPMD is caused by pathogenic variants in PABPN1 and inherited in either an autosomal dominant or an autosomal recessive manner [48].

d) Amish lethal microcephaly: It is characterized by microcephaly and early death. The occipitofrontal circumference is typically six to 12 SD below the mean, anterior and posterior fontanels are closed at birth, and facial features are distorted. The average life span is between five and six months.

Diagnosis is based on a tenfold increase in the levels of the urinary organic acid 2-ketoglutarate. SLC25A19 is the only gene known to be associated with Amish lethal microcephaly. All affected individuals within the old order Amish population are homozygous for the same single-base pair substitution [49-51].

e) Chronic progressive external ophthalmoplegia (CPE0): In a simpler case or when there is a maternal family history can result in large-scale single deletion of mtDNA that may only be detected in limited tissues (e.g., skeletal muscle). CPEO is sometimes complicated by mild proximal muscle weakness and dysphagia, and can be considered to lie on a spectrum of disease from pure CPEO to Kearns-Sayre syndrome phenotype. Some individuals with CPEO $(<20 \%)$ have a pathogenic single-nucleotide variant of mtDNA (e.g., m.3243A>G) [48].

f) Kearns-Sayre syndrome (KSS): A mtDNA deletion syndrome, is a multisystem disorder defined by the triad of onset before age 20 years, pigmentary retinopathy, and progressive external ophthalmoplegia (PEO). In addition, affected individuals have at least one of the following: cardiac conduction block, cerebrospinal fluid protein concentration greater than $100 \mathrm{mg} / \mathrm{dL}$, or cerebellar ataxia. Onset is usually in childhood. PEO, characterized by ptosis, paralysis of the extraocular muscles (ophthalmoplegia), and variably severe proximal limb weakness, is relatively benign [53].

g) BCS1L-related disorders: Pathogenic variants in BCS1L are associated with GRACILE (growth restriction, aminoaciduria, cholestasis, iron overload, lactic acidosis, and early death) syndrome, Bjørnstad syndrome (congenital profound hearing loss and pili torti), and an overlapping GRACILE syndrome-Bjørnstad syndrome phenotype. Children with the pure form of Bjørnstad syndrome have normal intellect.

The BCS1L protein is an assembly factor for complex III responsible for insertion of the Fe-S core into the complex. Affected children have a biochemical defect in complex III. The clinical scenario of a hepatoencephalopathy may appear similar to AHS at a single point in time, but mtDNA depletion is not part of the pathology described in those with BCS1L pathogenic variants [54,55]. h) Sc01-related disorders: Hepatic failure and severe encephalopathy have also been associated with biallelic pathogenic variants in SCO1 [56,57].

i) Neuronal ceroid-lipofuscinoses (NCLs): A group of inherited, neurodegenerative, lysosomal storage disorders characterized by progressive mental and motor deterioration, seizures, and early death. Visual loss is a feature of most forms. Phenotypes included in the NCLs that overlap with AHS are CLN1 disease, classic infantile NCL, CLN2 disease, and classic late infantile NCL $[58,59]$.

CLN1 disease: Children with CLN1 disease are normal at birth. Symptoms usually present acutely between ages 6 and 24 months. Initial signs include delayed development, myoclonic jerks and/or seizures, deceleration of head growth, and specific EEG changes. Affected infants develop retinal blindness and seizures by two years of age, followed by progressive mental deterioration. Pathogenic variants in PPT1 can be causative [60].

CLN2 disease: The first symptoms of CLN2 disease typically appear between age 2 and 4 years, usually starting with epilepsy, followed by regression of developmental milestones, dementia, ataxia, and extrapyramidal and pyramidal signs. Visual impairment typically appears between ages 4 and 6 years and rapidly progresses to blindness. Life expectancy ranges from age six years to greater than age 40 years. Pathogenic variants in PPT1, TPP1, CLN5, CLN6, and CLN8 can be causative [61].

j) MERRF: It is a multisystem disorder characterized by myoclonus (often the first symptom) followed by generalized epilepsy, ataxia, weakness, and dementia. Onset is usually in childhood, following normal early development. Common findings are hearing loss, short stature, optic atrophy, and cardiomyopathy with Wolff-Parkinson-White syndrome. Occasionally pigmentary retinopathy and lipomatosis are observed. MERRF is transmitted by maternal inheritance [62].

k) MELAS: It is a multisystem disorder with onset typically occurring in childhood. Early psychomotor development is usually normal. Onset of symptoms is often between 2-10 years. The most common initial symptoms are generalized tonic-clonic seizures, recurrent headaches, anorexia, and recurrent vomiting.

Seizures are often associated with stroke-like episodes of transient hemiparesis or cortical blindness. The cumulative residual effects of the stroke-like episodes gradually impair motor abilities, vision, and mentation, often by adolescence or young adulthood. Sensorineural hearing loss is common. MELAS is transmitted by maternal inheritance [63].

1) Other storage diseases: During infancy and early childhood, when there is history of multi organ involvement and drug resistant epileptiform discharges several mtDNA 
disorders such as atypical hexosaminidase A deficiency [64], Sandhoff disease [65], infantile sialidosis [66], and galactosialidosis [67], can be diagnosed by testing for lysosomal enzyme activity in the appropriate tissue.

\section{Discussion}

Polymerase gamma-related disorder widely known as POLG related disorder, comprises a spectrum of genetic mitochondrial disorders with overlapping phenotypes. Most common disorders are 1) Alpers syndrome; 2) myoclonic epilepsy myopathy sensory ataxia; 3) ataxia neuropathy spectrum; 4) autosomal recessive progressive external ophthalmoplegia; 5) Childhood myocerebrohepatopathy spectrum; 6) autosomal dominant progressive external ophthalmoplegia. No fixed clinical diagnostic criteria exists, hence diagnosis of the abovementioned disorders is based on clinical suspicion and genetic testing.

Treatment is solely conservative which may include occupational therapy, physical therapy, and speech therapy. Antiepileptic drugs (AEDs) may be used if seizure is a presenting symptom. However, any medications metabolized by hepatic enzymes should be used cautiously to avoid hepatotoxicity. Certain AEDs should not be used because of the risk of developing liver failure [68].

Avoidance of stress, infection, dehydration, fever, and anorexia can de-accelerate acute medical deterioration. Family members and caregivers should be properly educated about symptoms and precipitating factors. Survival of an individual with POLG related disorder largely depends on age at onset, presenting phenotype, and development of any secondary complications.

\section{Conclusion}

POLG-related disorders comprise a continuum of overlapping phenotypes that were clinically defined long before their molecular basis was known. Multiorgan system can be involved which can be confused with other storage diseases. Clinicians should bear in mind typical presenting features of each disorder which can aid in diagnosis along with the mutated gene or the enzyme involved. Not much treatment is available at this moment apart from the symptomatic management. More studies are required in future to understand the underlying molecular and pathogenic mechanisms which can be targeted as a primary therapeutic option.

\section{Disclosure}

The author has no relevant affiliations or financial involvement with any organization or entity with a financial interest in or financial conflict with the subject matter or materials discussed in the manuscript.

\section{Acknowledgement}

Special thanks to my supervisor Professor Dr. Huifang
Shang who gave initial ideas and supported me through this research study. I would also like to thank Dr. Swati lekha Roy Sarkar for her valuable feedback on the manuscript and literature screening.

\section{References}

1. Milone M, Benarroch EE, Wong LJ. POLG-related disorders: defects of the nuclear and mitochondrial genome interaction. Neurology. 2011; 77: 1847-1852.

PubMed: https://pubmed.ncbi.nlm.nih.gov/22084276/

2. Milone M, Massie R. Polymerase gamma 1 mutations: clinical correlations. Neurologist. 2010; 16: 84-91.

PubMed: https://pubmed.ncbi.nlm.nih.gov/20220442/

3. Wong LJ, Naviaux RK, Brunetti-Pierri N, Qing Zhang, Eric S Schmitt, et al. Molecular and clinical genetics of mitochondrial diseases due to POLG mutations. Hum Mutat. 2008; 29: E150-E172.

PubMed: https://pubmed.ncbi.nlm.nih.gov/18546365/

4. Darin N, Oldfors A, Moslemi AR, Holme E, Tulinius M. The incidence of mitochondrial encephalomyopathies in childhood: clinical features and morphological, biochemical, and DNA abnormalities. Ann Neurol. 2001; 49: 377-383.

PubMed: https://pubmed.ncbi.nlm.nih.gov/11261513/

5. Rahman S, Copeland WC. POLG-related disorders and their neurological manifestations. Nat Rev Neurol. 2019; 15: 40-52. PubMed: https://pubmed.ncbi.nlm.nih.gov/30451971/

6. Saneto RP, Cohen BH, Copeland WC, Naviaux RK. AlpersHuttenlocher syndrome. Pediatr Neurol. 2013; 48: 167-178. PubMed: https://pubmed.ncbi.nlm.nih.gov/23419467/

7. Hikmat O, Tzoulis C, Knappskog PM, Johansson S, Boman H, et al ADCK3 mutations with epilepsy, stroke-like episodes and ataxia: a POLG mimic? Eur J Neurol. 2016; 23: 1188-1194. PubMed: https://pubmed.ncbi.nlm.nih.gov/27106809/

8. Antozzi C, Franceschetti S, Filippini G, B Barbiroli, M Savoiardo, et al. Epilepsia partialis continua associated with NADH-coenzyme $Q$ reductase deficiency. J Neurol Sci. 1995; 129: 152-161. PubMed: https://pubmed.ncbi.nlm.nih.gov/7608730/

9. Mameniškienè $R$, Wolf $P$. Epilepsia partialis continua: A review. Seizure. 2017; 44: 74-80.

PubMed: https://pubmed.ncbi.nlm.nih.gov/28029552/

10. Zhou Q, Lin Y, Ye J, Li L, Hu N, et al. Homozygous TBC1D24 Mutation in a Case of Epilepsia Partialis Continua. Front Neurol. 2018; 8: 750. PubMed: https://pubmed.ncbi.nlm.nih.gov/29416524/

11. Dai LF, Fang F, Liu ZM, Shen DM, Ding $\mathrm{CH}$, et al. Zhonghua Er Ke Za Zhi. 2019; 57: 211-216.

PubMed: https://pubmed.ncbi.nlm.nih.gov/30818899/

12. El-Hattab AW, Scaglia F. Mitochondrial DNA depletion syndromes: review and updates of genetic basis, manifestations, and therapeutic options. Neurotherapeutics. 2013; 10: 186-198.

PubMed: https://pubmed.ncbi.nlm.nih.gov/23385875/

13. Nogueira C, Almeida LS, Nesti C, Pezzini I, Videira A, et al. Syndromes associated with mitochondrial DNA depletion. Ital J Pediatr. 2014; 40: 34 PubMed: https://pubmed.ncbi.nlm.nih.gov/24708634/

14. Saada A, Shaag A, Mandel H, Nevo Y, Eriksson S, et al. Mutant mitochondrial thymidine kinase in mitochondrial DNA depletion myopathy. Nat Genet. 2001; 29: 342-344.

PubMed: https://pubmed.ncbi.nlm.nih.gov/11687801/

15. Spinazzola A, Invernizzi F, Carrara F, Lamantea E, Donati A, et al. Clinical and molecular features of mitochondrial DNA depletion syndromes. J Inherit Metab Dis. 2009; 32: 143-158.

PubMed: https://pubmed.ncbi.nlm.nih.gov/19125351/

16. Buchaklian AH, Helbling D, Ware SM, Dimmock DP. Recessive 
deoxyguanosine kinase deficiency causes juvenile onset mitochondria myopathy. Mol Genet Metab. 2012; 107: 92-94.

PubMed: https://pubmed.ncbi.nlm.nih.gov/22622127/

17. Bourdon A, Minai L, Serre V, Jean-Philippe J, Sarzi E, et al. Mutation of RRM2B, encoding p53-controlled ribonucleotide reductase (p53R2) causes severe mitochondrial DNA depletion. Nat Genet. 2007; 39: 776-780

PubMed: https://pubmed.ncbi.nlm.nih.gov/17486094/

18. Lesko N, Naess K, Wibom R, Solaroli N, Nennesmo I, et al. Two novel mutations in thymidine kinase-2 cause early onset fatal encephalomyopathy and severe mtDNA depletion. Neuromuscul Disord. 2010; 20: 198-203.

PubMed: https://pubmed.ncbi.nlm.nih.gov/20083405/

19. ElpelegO,MillerC,HershkovitzE,Bitner-GlindziczM,Bondi-Rubinstein G et al. Deficiency of the ADP-forming succinyl-CoA synthase activity is associated with encephalomyopathy and mitochondrial DNA depletion. Am J Hum Genet. 2005; 76: 1081-1086.

$\mathrm{P}$ u b M e d: https://pubmed.ncbi.nlm.nih.gov/15877282/

20. Ostergaard E, Christensen E, Kristensen E, Mogensen B, Duno M, et al. Deficiency of the alpha subunit of succinate-coenzyme A ligase causes fatal infantile lactic acidosis with mitochondrial DNA depletion. Am J Hum Genet. 2007; 81: 383-387.

PubMed: https://www.ncbi.nlm.nih.gov/pmc/articles/PMC1950792/

21. Sarzi E, Bourdon A, Chrétien D, Zarhrate $M$, Corcos $J$, et al. Mitochondrial DNA depletion is a prevalent cause of multiple respiratory chain deficiency in childhood. J Pediatr. 2007; 150: 531-534.e5346. PubMed: https://pubmed.ncbi.nlm.nih.gov/17452231/

22. Mandel H, Szargel R, Labay V, Elpeleg O, Saada A, et al. The deoxyguanosine kinase gene is mutated in individuals with depleted hepatocerebral mitochondrial DNA [published correction appears in Nat Genet. 2001; 29: 337-341.

PubMed: https://pubmed.ncbi.nlm.nih.gov/11687800/

23. Spinazzola A, Viscomi C, Fernandez-Vizarra E, Carrara F, D'Adamo $P$ et al. MPV17 encodes an inner mitochondrial membrane protein and is mutated in infantile hepatic mitochondrial DNA depletion. Nat Genet. 2006; 38: 570-575.

PubMed: https://pubmed.ncbi.nlm.nih.gov/16582910/

24. Zhang S, Li FY, Bass HN, Pursley A, Schmitt ES, et al. Application of oligonucleotide array $\mathrm{CGH}$ to the simultaneous detection of a deletion in the nuclear TK2 gene and mtDNA depletion. Mol Genet Metab. 2010; 99: 53-57.

PubMed: https://pubmed.ncbi.nlm.nih.gov/19815440/

25. Nishino I, Spinazzola A, Hirano M. Thymidine phosphorylase gene mutations in MNGIE, a human mitochondrial disorder. Science. 1999; 283: 689-692.

PubMed: https://pubmed.ncbi.nlm.nih.gov/9924029/

26. Shaibani A, Shchelochkov OA, Zhang S, Katsonis $\mathrm{P}$, Lichtarge $\mathrm{O}$, et al. Mitochondrial neurogastrointestinal encephalopathy due to mutations in RRM2B. Arch Neurol. 2009; 66: 1028-1032.

PubMed: https://pubmed.ncbi.nlm.nih.gov/19667227/

27. Dimmock DP, Zhang Q, Dionisi-Vici C, Carrozzo R, Shieh J, et al. Clinical and molecular features of mitochondrial DNA depletion due to mutations in deoxyguanosine kinase. Hum Mutat. 2008; 29: 330-331. PubMed: https://pubmed.ncbi.nlm.nih.gov/18205204/

28. Qualls C, Kornfeld M, Joste N, Ali AM, Appenzeller O. MPV17-related hepatocerebral mitochondrial DNA depletion syndrome (MPV17-NNH) revisited. eNeurologicalSci. 2016; 2: 8-13.

PubMed: https://pubmed.ncbi.nlm.nih.gov/29473055/

29. El-Hattab AW, Wang J, Dai H, Almannai M, Staufner C, et al. MPV17related mitochondrial DNA maintenance defect: New cases and review of clinical, biochemical, and molecular aspects. Hum Mutat. 2018; 39 : 461-470.

PubMed: https://pubmed.ncbi.nlm.nih.gov/29282788/
30. Baumann M, Schreiber H, Schlotter-Weigel B, Löscher WN, Stucka R, et al. MPV17 mutations in juvenile- and adult-onset axonal sensorimotor polyneuropathy. Clin Genet. 2019; 95: 182-186.

PubMed: https://pubmed.ncbi.nlm.nih.gov/30298599/

31. Couser NL, Marchuk DS, Smith LD, Arreola A, Kaiser-Rogers KA, et al. Co-occurring Down syndrome and SUCLA2-related mitochondrial depletion syndrome. Am J Med Genet A. 2017; 173: 2720-2724. PubMed: https://pubmed.ncbi.nlm.nih.gov/28749033/

32. Ballout RA, AI Alam C, Bonnen PE, Huemer M, El-Hattab AW, Shbarou R. FBXL4-Related Mitochondrial DNA Depletion Syndrome 13 (MTDPS13): A Case Report With a Comprehensive Mutation Review. Front Genet. 2019; 10: 39.

PubMed: https://pubmed.ncbi.nlm.nih.gov/30804983/

33. Landsverk ML, Zhang VW, Wong LC, Andersson HC. A SUCLG1 mutation in a patient with mitochondrial DNA depletion and congenital anomalies. Mol Genet Metab Rep. 2014; 1: 451-454. PubMed: https://pubmed.ncbi.nlm.nih.gov/27896121/

34. Pitceathly RD, Smith C, Fratter C, He L, Craig K, et al. Adults with RRM2B-related mitochondrial disease have distinct clinical and molecular characteristics. Brain. 2012; 135: 3392-3403. PubMed: https://pubmed.ncbi.nlm.nih.gov/23107649/

35. Madhok J, Leong J, Cohn J. Anesthetic Considerations for Liver Transplantation in a Patient with Mitochondrial Neurogastrointestinal Encephalopathy Syndrome. Cureus. 2019; 11: e5038. PubMed: https://pubmed.ncbi.nlm.nih.gov/31501730/

36. Filosto M, Cotti Piccinelli S, Caria F, Cassarino SG, Baldelli E, et al. Mitochondrial Neurogastrointestinal Encephalomyopathy (MNGIEMTDPS1). J Clin Med. 2018; 7: 389

PubMed: https://pubmed.ncbi.nlm.nih.gov/30373120/

37. Wang HF, Wang J, Wang YL, Jian-Jian F, Gui-Lin M, et al. A novel thymidine phosphorylase mutation in a Chinese MNGIE patient. Acta Neurol Belg. 2017; 117: 259-267.

PubMed: https://pubmed.ncbi.nlm.nih.gov/27709505/

38. Yong FL, Wang CW, Tan KS. MicroRNA expression profile of a Malaysian Bajau family with familial mitochondrial neurogastrointestinal encephalomyopathy. Genet Mol Res. 2015; 14: 13172-13183. PubMed: https://pubmed.ncbi.nlm.nih.gov/26535630/

39. Oskoui M, Davidzon G, Pascual J, Erazo R, Gurgel-Giannetti J, et al Clinical spectrum of mitochondrial DNA depletion due to mutations in the thymidine kinase 2 gene. Arch Neurol. 2006; 63: 1122-1126. PubMed: https://pubmed.ncbi.nlm.nih.gov/16908738/

40. Martín-Hernández E, García-Silva MT, Quijada-Fraile P, RodríguezGarcía ME, Rivera $\mathrm{H}$, et al. Myopathic mtDNA Depletion Syndrome Due to Mutation in TK2 Gene. Pediatr Dev Pathol. 2017; 20: 416-420. PubMed: https://pubmed.ncbi.nlm.nih.gov/28812460/

41. Wang J, Kim E, Dai H, Stefans V, Vogel H, et al. Clinical and molecular spectrum of thymidine kinase 2-related mtDNA maintenance defect. Mol Genet Metab. 2018; 124: 124-130.

PubMed: https://pubmed.ncbi.nlm.nih.gov/29735374/

42. El-Hattab AW, Craigen WJ, Scaglia F. Mitochondrial DNA maintenance defects. Biochim Biophys Acta Mol Basis Dis. 2017; 1863: 1539-1555. PubMed: https://pubmed.ncbi.nlm.nih.gov/28215579/

43. Matic S, Jiang M, Nicholls TJ, Dirksen-Schwanenland C, Polosa PL, et al Mice lacking the mitochondrial exonuclease MGME1 accumulate mtDNA deletions without developing progeria. Nat Commun. 2018; 9: 1202. PubMed: https://pubmed.ncbi.nlm.nih.gov/29572490/

44. Rusecka J, Kaliszewska M, Bartnik E, Tońska K. Nuclear genes involved in mitochondrial diseases caused by instability of mitochondrial DNA. J Appl Genet. 2018; 59: 43-57.

PubMed: https://pubmed.ncbi.nlm.nih.gov/29344903/

45. Baertling F, Rodenburg RJ, Schaper J, Smeitink JA, Koopman WJH, et al. A guide to diagnosis and treatment of Leigh syndrome. J Neurol Neurosurg Psychiatry. 2014; 85: 257-265.

PubMed: https://pubmed.ncbi.nlm.nih.gov/23772060/ 
46. Suomalainen A, Majander A, Wallin M, Setälä K, Kontula K, et al. Autosomal dominant progressive external ophthalmoplegia with multiple deletions of mtDNA: clinical, biochemical, and molecular genetic features of the 10q-linked disease. Neurology. 1997; 48: 1244-1253. PubMed: https://pubmed.ncbi.nlm.nih.gov/9153451/

47. Kiechl $S$, Horváth R, Luoma $\mathrm{P}$, Kiechl-Kohlendorfer $U$, WallacherScholz B, et al. Two families with autosomal dominant progressive external ophthalmoplegia. J Neurol Neurosurg Psychiatry. 2004; 75: 1125-1128.

PubMed: https://pubmed.ncbi.nlm.nih.gov/15258213/

48. Galimberti V, Tironi R, Lerario A, Scali M, Del Bo R, et al. Value of insoluble PABPN1 accumulation in the diagnosis of oculopharyngeal muscular dystrophy. Eur J Neurol. 2020; 27: 709-715.

PubMed: https://pubmed.ncbi.nlm.nih.gov/31769567/

49. Kelley RI, Robinson D, Puffenberger EG, Strauss KA, Morton DH Amish lethal microcephaly: a new metabolic disorder with severe congenital microcephaly and 2-ketoglutaric aciduria. Am J Med Genet. 2002; 112: 318-326.

PubMed: https://pubmed.ncbi.nlm.nih.gov/12376931/

50. Siu VM, Ratko S, Prasad AN, Prasad C, Rupar CA. Amish microcephaly: Long-term survival and biochemical characterization. Am J Med Genet A. 2010; 152A: 1747-1751.

51. Bottega R, Perrone MD, Vecchiato K, Taddio A, Sabui S, et al Functional analysis of the third identified SLC25A19 mutation causative for the thiamine metabolism dysfunction syndrome 4. J Hum Genet. 2019; 64: 1075-1081.

PubMed: https://www.ncbi.nlm.nih.gov/pmc/articles/PMC6886476/

52. Heighton JN, Brady LI, Sadikovic B, Bulman DE, Tarnopolsky MA. Genotypes of chronic progressive external ophthalmoplegia in a large adult-onset cohort. Mitochondrion. 2019; 49: 227-231.

PubMed: https://pubmed.ncbi.nlm.nih.gov/31521625/

53. Saldaña-MartínezA, MuñozML,Pérez-RamírezG, etal.Wholesequence of the mitochondrial DNA genome of Kearns Sayre Syndrome patients: Identification of deletions and variants. Gene. 2019; 688: 171-181. PubMed: https://pubmed.ncbi.nlm.nih.gov/30528267/

54. Oláhová M, Ceccatelli Berti C, Collier JJ, Alston CL, Jameson E, et al. Molecular genetic investigations identify new clinical phenotypes associated with BCS1L-related mitochondrial disease. Hum Mol Genet. 2019; 28: 3766-3776.

PubMed: https://pubmed.ncbi.nlm.nih.gov/31435670/

55. Baker RA, Priestley JRC, Wilstermann AM, Reese KJ, Mark PR. Clinical spectrum of BCS1L Mitopathies and their underlying structural relationships. Am J Med Genet A. 2019; 179: 373-380.

PubMed: https://pubmed.ncbi.nlm.nih.gov/30582773/

56. Valnot I, Osmond S, Gigarel N, Mehaye B, Amiel J, et al. Mutations of the SCO1 gene in mitochondrial cytochrome $c$ oxidase deficiency with neonatal-onset hepatic failure and encephalopathy. Am J Hum Genet. 2000; 67: 1104-1109.

PubMed: https://pubmed.ncbi.nlm.nih.gov/11013136/

57. Hlynialuk CJ, Ling B, Baker ZN, Cobine PA, Yu LD, et al. The Mitochondrial Metallochaperone SCO1 Is Required to Sustain
Expression of the High-Affinity Copper Transporter CTR1 and Preserve Copper Homeostasis. Cell Rep. 2015; 10: 933-943.

PubMed: https://pubmed.ncbi.nlm.nih.gov/25683716/

58. Nelvagal HR, Lange J, Takahashi K, Tarczyluk-Wells MA, Cooper JD. Pathomechanisms in the neuronal ceroid lipofuscinoses. Biochim Biophys Acta Mol Basis Dis. 2020; 1866: 165570.

PubMed: https://pubmed.ncbi.nlm.nih.gov/31678162/

59. Butz ES, Chandrachud U, Mole SE, Cotman SL. Moving towards a new era of genomics in the neuronal ceroid lipofuscinoses. Biochim Biophys Acta Mol Basis Dis. 2020; 1866: 165571. PubMed: https://pubmed.ncbi.nlm.nih.gov/31678159/

60. Hawkins-Salsbury JA, Cooper JD, Sands MS. Pathogenesis and therapies for infantile neuronal ceroid lipofuscinosis (infantile CLN1 disease). Biochim Biophys Acta. 2013; 1832: 1906-1909.

PubMed: https://pubmed.ncbi.nlm.nih.gov/23747979/

61. Nickel M, Simonati A, Jacoby D, Lezius S, Kilian D, et al. Disease characteristics and progression in patients with late-infantile neuronal ceroid lipofuscinosis type 2 (CLN2) disease: an observational cohort study. Lancet Child Adolesc Health. 2018; 2: 582-590. PubMed: https://pubmed.ncbi.nlm.nih.gov/30119717/

62. Shoffner JM, Lott MT, Lezza AM, Seibel P, Ballinger SW, et al. Myoclonic epilepsy and ragged-red fiber disease (MERRF) is associated with a mitochondrial DNA tRNA(Lys) mutation. Cell. 1990; 61: 931-937. PubMed: https://pubmed.ncbi.nlm.nih.gov/2112427/

63. Ikeda T, Osaka H, Shimbo H, Tajika M, Yamazaki M, et al. Mitochondrial DNA 3243A>T mutation in a patient with MELAS syndrome. Hum Genome Var. 2018; 5: 25.

PubMed: https://pubmed.ncbi.nlm.nih.gov/30210801/

64. Rozenberg R, Kok F, Burin MG, Sá Miranda MC, Vasques C, et al. Diagnosis and molecular characterization of non-classic forms of TaySachs disease in Brazil. J Child Neurol. 2006; 21: 540-544. PubMed: https://pubmed.ncbi.nlm.nih.gov/16948947/

65. Liu M, Huang D, Wang H, Zhao L, Wang Q, Chen X. Clinical and Molecular Characteristics of Two Chinese Children with Infantile Sandhoff Disease and Review of the Literature. J Mol Neurosci. 2020; 70: 481-487.

PubMed: https://pubmed.ncbi.nlm.nih.gov/31919734/

66. Khan A, Sergi C. Sialidosis: A Review of Morphology and Molecular Biology of a Rare Pediatric Disorder. Diagnostics (Basel). 2018; 8: 29. PubMed: https://pubmed.ncbi.nlm.nih.gov/29693572/

67. Sláma T, Garbade SF, Kölker S, Hoffmann GF, Ries M. Quantitative natural history characterization in a cohort of 142 published cases of patients with galactosialidosis-A cross-sectional study. J Inherit Metab Dis. 2019; 42: 295-302.

PubMed: https://pubmed.ncbi.nlm.nih.gov/30693535/

68. Cohen BH, Chinnery PF, Copeland WC. POLG-Related Disorders. In: GeneReviews at GeneTests: Medical Genetics Information Resource (database online). Copyright University of Washington, Seattle. 19932018.

PubMed: http://www.ncbi.nlm.nih.gov/books/NBK26471/ 